\title{
Investigação de parasitos contaminantes em quadra de areia no município de Gaspar (SC)
}

\author{
Marcelo Alberto Elias \\ Instituto Federal de Santa Catarina - IFSC \\ Departamento Ensino, Pesquisa e Extensão \\ (marcelo.elias@ifsc.edu.br) \\ João Elias de Almeida \\ Instituto Federal de Santa Catarina - IFSC \\ Estudante Curso Técnico Integrado em Química \\ (joao.e.a@hotmail.com)
}

\begin{abstract}
Resumo: 0 município de Gaspar, com aproximadamente 65 mil habitantes, necessita de áreas de lazer para a população aproveitar seu tempo livre. Com isso, o município disponibiliza quadras de esportes, academias ao ar livre, ginásios de esportes, quadras de areia, pista de skate, entre outros. Porém, como há um grande uso dessas, animais também habitam esses locais e, com isso, o risco de contrair doenças, principalmente parasitológicas, é elevado. Com base nisso, foram realizadas análises da quadra de areia localizada no bairro Bela Vista, próximo ao campus do Instituto Federal de Santa Catarina (IFSC). Foi realizada uma pesquisa com cunho qualitativo exploratória, em que foram utilizados métodos de Hoffman e Rugai, ambos modificados, que são métodos clássicos para a investigação de parasitos em areia. Os 5 pontos coletados para a análise foram determinados em método de zigue-zague, que visa um melhor espaço amostral, aumentando a homogeneidade da amostra, e com no máximo 10 centímetros de profundidade. Os materiais utilizados para as análises foram equipamentos de laboratório, béqueres, espátulas, água da torneira, funil simples, tubos de ensaio, centrífuga, lâminas e lamínulas, lugol como corante, além de microscópio com lentes objetivas de 10x e 40x. Os resultados obtidos foram positivos para a presença de ovos dos parasitos Ascaris lumbricoides e Trichuris trichiura, assim como larvas de Ancilostomídeos e Strongiloides sp. Com base nos resultados encontrados, visto que os parasitos presentes no local são contaminantes dos seres humanos, fica a importância de levar esses dados para os órgãos públicos competentes, para uma possível limpeza e fechamento do local para evitar a presença de animais errantes. Outro ponto importante é a divulgação desse resultado com a comunidade que utiliza a quadra e, por fim, os métodos Hoffman e Rugai demostraram-se como eficazes para investigações e análises básicas de parasitos.
\end{abstract}

PALAVRAS-CHAVE: Parasitos em areia; Doenças negligenciadas; Saúde pública.

\section{Investigation of contaminant parasites in sand courts in the Gaspar city (SC)}

Abstract: The Gaspar city, with approximately 65 thousand inhabitants, needs leisure areas for the population to enjoy their free time. Thereby, the county offers sports courts, outdoor gyms, sports gyms, sand courts, skate track, among others. However, since there is a great use of these, animals also inhabit these places and, with this, the risk of contracting mainly parasitological diseases is high. Based on this, analyzes of the sand court located in the neighborhood Bela Vista, near the campus of the Federal Institute of Santa Catarina (IFSC), were performed. An exploratory qualitative research was carried out, using methods of Hoffman and Rugai, both modified, which are classic methods for the investigation of sand parasites. The 5 points collected for the analysis were determined in a zigzag method, which aims at a better sample space, increasing the homogeneity of the sample, and with a maximum depth of 10 centimeters. The materials used for the analysis were laboratory equipment, beakers, spatulas, tap water, simple funnel, test tubes, centrifuge, laminates and coverslips, lugol as a dye, as well as a microscope with objective lenses of 10x and 40x. The results obtained were positive for the presence of eggs of the parasites Ascaris lumbricoides and Trichuris trichiura, as well as larvae of Hookworms and Strongiloides sp. Based on the results found, since the parasites present in the 
place are contaminants of humans, it is important to take these data to the competent public agencies for a possible cleaning and closure of the place to avoid the presence of wandering animals. Another important point is the dissemination of this result to the community that uses the court and, finally, the methods Hoffman and Rugai have proved to be effective for basic investigations and analysis of parasites.

Keywords: sand parasites; neglected diseases; public health.

\section{INTRODUÇÃO}

O município de Gaspar situa-se no chamado coração do vale do rio Itajaí-açu, com uma área total de $386,776 \mathrm{~km}^{2}$, sendo essa área na sua maioria rural e, portanto, pouco habitada. Com uma população estimada, em 2013 pelo IBGE, em 64.024 mil habitantes, Gaspar necessita de áreas de lazer para a sua população e conta com instalações como: quadras de esportes, pistas de skate, quadras de areia, academias ao ar livre, parques infantis, clube para a prática de aeromodelismo, além de contar com um local para a prática de voos livres (GASPAR, 2014).

No entanto, as principais instalações utilizadas pela população são os parques infantis, as quadras de esportes e as academias ao ar livre. Em algumas academias há também quadras de areia ao lado, como é o caso do bairro Bela Vista. Onde há academia, quadra de areia e ginásio, encontra-se também uma Unidade Básica de Saúde, o que contribui para a utilização do espaço público.

Porém, praças e áreas de recreação públicas ou privadas que contêm areia têm um grande potencial para a proliferação de parasitos, principalmente em espaços públicos em que não há isolamento para animais, nem controle de entrada e saída, além da falta de limpeza e desinfecção periódica desses locais. A contaminação desses locais ocorre, na maioria das vezes, por animais domésticos, como cães e gatos (PINHEIRO, 2016).

Cães e gatos com acesso aos locais de recreação contaminam o solo, eliminando até 15.000 ovos de parasitas por grama de fezes, e estes permanecem viáveis por longo período no ambiente, expondo a população humana ao risco de infecção e desenvolvimento de doenças (ALVES, 2014).

Os parasitos intestinais podem causar danos aos seus portadores, como a obstrução intestinal (Ascaris lumbricoides), desnutrição (Ascaris lumbricoides e Trichuris trichiura), anemia por deficiência de ferro (ancilostomídeos) e quadros de 
diarreia e má absorção de nutrientes (Entamoeba histolytica e Giardia lamblia), sendo que as manifestações clínicas são usualmente proporcionais à carga parasitária albergada no indivíduo (PINHEIRO, 2015).

Vários parasitos podem ser encontrados na areia, trazendo perigos para a saúde humana, entre eles podemos citar os Ascaris lumbricoides, Toxocara canis, Strongyloides stercoralis, Ancylostoma spp. e o Trichuris trichiura (REY, 2008). O Ascaris lumbricoides também é conhecido como lombrigas ou popularmente como "bichas". Localizam-se preferencialmente no duodeno e no jejuno, onde produzem um quadro clínico variado, denominado ascaridíase.

Ovos de Ascaris lumbricoides podem ser ingeridos com os alimentos ou a partir das mãos sujas, mas, também, ser aspirados e depois deglutidos com o muco das vias respiratórias (SILVA ET AL., 2013). Segundo o mesmo autor, no aspecto epidemiológico os parasitos Ancilostomídeos (família Ancylostomatidae) têm uma contaminação global, mas para que tenha a existência de infecção depende das condições ecológicas estritamente locais e circunscritas.

Segundo Portal (2013), os Balantidium sp. são uma espécie de protozoário que existe no intestino grosso dos suínos e algumas podem parasitar os humanos. Só podem ser contraídos pelos humanos por meio de alguma lesão existente na pele. Dotado de produzir hialuronidase, pode aumentar a lesão, ocasionando necrose e úlcera local; tais lesões, como a própria sintomatologia, são semelhantes às que ocorrem na amebíase, visto que o paciente, nesse caso, apresenta diarreia, gases, dor abdominal, anorexia, fraqueza e, às vezes, febre.

Segundo Silva (2013), não existem parâmetros indicativos por conta da incerteza cientifica, não há controle sanitário para areias de praia e parques. Porém, por meio da resolução n 20, de 18 de junho de 1986, há recomendação aos órgãos municipais a avaliação das condições parasitológicas.

Alguns estudos abordam seu potencial zoonótico, enteroparasitose em crianças e suas consequências como a presença de parasitoses intestinais, além de pesquisas voltadas a falta de saneamento básico (PINHEIRO, 2015; PINHEIRO, 2016).

Existem outros que tratam da presença de parasitos em vários lugares, como em praças públicas, por fezes de animais em praças públicas, na praia, além de pesquisas sobre parasitos em caixas de areia de creches públicas, em creches públicas e privadas (FIGUEREDO et al., 2011). 
Alves (2014) observou a presença de parasitos na areia da praia e, para isso, usou os métodos Rugai e Hoffman, ambos modificados, para análise.

Com base nisso, o presente projeto tem como objetivo analisar a areia da área de lazer localizada ao lado do IFSC - Campus Gaspar, para identificar a existência de ovos ou larvas de parasitos. Caso haja, será recomendada uma futura limpeza ou desinfecção da área e uma conscientização da população do bairro junto à prefeitura para manter essas áreas limpas e livres de parasitos.

\section{METODOLOGIA}

Essa pesquisa é de cunho qualitativo e os métodos utilizados para a análise das amostras são o Rugai modificado e Hoffman (ALVES, 2014). Serão utilizados em função do baixo custo que estes métodos dispõem, também devido à praticidade para a coleta dos dados e sua simplicidade para a realização.

O ambiente de pesquisa foi uma área de lazer localizada no bairro Bela Vista, no município de Gaspar, no estado Santa Catarina, no mês de julho de 2016. A área é utilizada pela comunidade, inclusive por muitas crianças, para a prática de atividades físicas e esportes.

Os pontos a serem analisados foram definidos em zigue-zague, pois objetiva utilizar melhor o espaço amostral, a fim de proporcionar uma melhor busca por dados qualitativos, diminuindo a probabilidade de erro.

As amostras foram coletadas com uma profundidade de 10 centímetros, em cinco pontos diferentes. Foram coletados em cada ponto cerca de 300 gramas de material e colocados em sacos plásticos estéreis e descartáveis, com informações de cada ponto.

No dia da coleta foi possível observar a presença de cães e fezes de animais no local. Os materiais utilizados na coleta foram sacos plásticos estéreis e descartáveis, espátulas para a coleta do material, etiquetas de identificação e caixa plástica para o armazenamento e o transporte das amostras.

Para as análises das amostras, foram utilizados béqueres de $50 \mathrm{ml}, 250 \mathrm{ml}$ e $1000 \mathrm{ml}$, luvas de procedimento, banho-maria, gaze, tubos de ensaio, bastões de vidro, centrífuga, água, papel toalha, pipeta de Pasteur, lâminas e lamínulas. 
Método Rugai Modificado

As amostras coletadas foram preparadas no laboratório de biologia e microbiologia do IFSC - Campus Gaspar e analisadas pelo método Rugai modificado. As amostras foram homogeneizadas e colocaram-se duas espátulas do material em quatro gazes separadas e imersas em béqueres de $50 \mathrm{ml}$ com água a $45^{\circ}$ Celsius por uma hora, a fim de desprender as estruturas possíveis de parasitos da areia.

Descartou-se o sobrenadante, colocou-se o sedimento em tubos de ensaio e, posteriormente, na centrífuga a 2000rpm por dois minutos. Lâminas e lamínulas foram preparadas, coradas com lugol e analisadas no microscópio com lentes objetivas de 10x e 40x para verificar a existência de ovos, larvas e/ou outras estruturas de parasitos.

As modificações realizadas no estudo foram quatro trouxas de gaze; por não conter cálices de sedimentação foram usados béqueres de $50 \mathrm{ml}$; as amostras não foram reanalisadas após os períodos de 48 e 72 horas.

\section{Método Hoffman Modificado}

Colocaram-se cerca de 250 gramas de material em Béquer de 1000ml. Completou-se o volume de $1000 \mathrm{ml}$ com água a $45^{\circ}$ Celsius e misturou-se vigorosamente com bastão de vidro. Aguardou-se uma hora a fim de todas as estruturas de parasitos a serem analisadas se desprendessem da areia.

A suspensão foi filtrada através de gaze, recolhendo o filtrado num béquer de 250ml. Centrifugou-se a suspensão a 2000rpm por dois minutos. Com uma pipeta de Pasteur, retiraram-se duas gotas do sedimento que foi depositado na lamínula, corado com lugol e observado no microscópio com lentes objetivas de 10x e 40x e repetido sem lugol para visualizar a motilidade de larvas e outros parasitos. Todas as analisem de areia foram feitas em duplicata nos dois métodos de análises utilizados e conferência do orientador.

As modificações realizadas no estudo foram: através do uso de um béquer de $1000 \mathrm{ml}$, com isso pode-se utilizar mais material para análise; o filtrado foi recolhido num béquer de $50 \mathrm{ml}$, pois não se tem cálice de decantação no laboratório; centrifugação do filtrado a 2000rpm por dois minutos; e as amostras não foram reanalisadas após os períodos de 48 e 72 horas. 


\section{RESULTADOS E DISCUSSÃO}

Neste trabalho, foi observado a presença de ovos em todas as 50 amostras analisadas. O resultado encontrado é compatível com o encontrado por outros autores como Silva et al. (2013), Figueredo et al. (2012) e Pinheiro (2016), que também encontraram ovos e parasitos na areia.

Os ovos encontrados nas amostras pertencem aos parasitos Ascaris lumbricoides e ao Trichuris trichiura, que são causadores de doenças em humanos, o primeiro podendo causar ascaridíase, popularmente conhecida como lombriga, que é a doença intestinal mais disseminada no mundo, com mais ocorrência em países subdesenvolvidos, e localizam-se de preferência no duodeno e no jejuno, onde produzem um quadro clínico variado (ALVES, 2014).

Existem três tipos de contaminação pelos ovos de Ascaris lumbricoides, sendo por meio de alimentos, pelas mãos sujas ou aspirado pelas vias aéreas. Em geral, apenas uma em cada seis pessoas parasitadas apresenta manifestações clínicas decorrentes do parasitismo (PINHEIRO, 2015).

O homem é a única fonte de infecção, sendo as crianças em idade escolar e pré-escolar as principais poluidoras do meio e, também, a população de mais alto risco; pelo hábito de defecarem no chão, o local peridomicílio mantém-se sempre rico em ovos (REY, 2008).

Cerca de $45 \%$ das oclusões hospitalares por bolos de Ascaris lumbricoides ocorrem em crianças com menos de dois anos de idade, pelo motivo de brincarem normalmente em lugares com contato com o solo e levarem as mãos sujas diretamente à boca. Ela também é o parasito mais comum em países subdesenvolvidos, sendo uns dos principais motivos a falta de saneamento básico, habito poucos higiênicos, além do crescimento populacional desordenado.

Já os ovos de Trichuris trichiura, por sua vez, causam a Tricuríase, que é uma doença parecida com a anterior, porém seu nematódeo atinge no máximo quatro centímetros, e fica preso na parede do intestino grosso e a maioria dos infectados têm poucos sintomas da doença. Segundo Pinheiro (2016), a Tricuríase tem distribuição semelhante à da Ascaridíase e atinge, principalmente, crianças na região amazônica e faixa litorânea, com idade pré-escolar e com sintomas da doença mais agudos.

As crianças também são as mais responsáveis pela transmissão da doença. 
O peridomicílio é a área mais afetada, devido à poluição fecal do solo, onde as condições de saneamento e de higiene são precárias (REY, 2008).

Segundo Silva et al. (2014), os ovos Trichuris trichiura se desidratam mais facilmente em relação aos ovos de Ascaris lumbricoides, portanto, as áreas com maior risco de contaminação são em lugares com solo úmido e sombreado.

Já as larvas encontradas foram as das familias Ancilostomideos e Strongiloides sp. A familia dos Ancilostomideos infecta os seres humanos com duas espécies, sendo elas Ancylostoma duodenale e Necator americanos. Existem duas espécies que infectam cães e gatos, porém elas não conseguem completar sua evolução nos seres humanos (REY, 2008).

Ainda segundo o mesmo autor, as duas produzem praticamente o mesmo quadro clinico e quando atinge o estado adulto, os vermes se prendem ao intestino delgado, sugando o sangue do infectado. Os sintomas característicos de ancilostomose são dores abdominais após a chegada do parasito ao intestino, dor epigástrica, diminuição de apetite, indigestão, cólica, indisposição, náuseas, vômitos, em alguns casos pode haver diarreia sanguinolenta (ou não), e constipação (raramente).

Segundo Rey (2008), apesar de sua distribuição ser global, ele precisa de circunstâncias bem especificas para sobreviver. Os parasitos não se desenvolvem em locais com umidade inferior a $90 \%$ e os raios solares matam os embriões, assim sendo o solo arenoso, que preserva a umidade e a aeração, quando em matéria orgânica, contribui para o desenvolvimento dos estágios de vida livre, como próximo a residências.

A larva de Strongiloides sp. é a causadora da doença estrongiloidíase. O Strongyloides stercoralis costuma habitar o intestino delgado dos indivíduos infectados e, na maioria dos pacientes com sistema imunológico sadio, não provoca sintomas relevantes (PINHEIRO, 2015).

Os sintomas mais importantes são os digestivos, variando com a carga parasitária, como desconforto abdominal, cólicas, dores vagas ou imitando úlcera péptica, surtos diarreicos, anorexia, náuseas e vômitos. É mais comum em regiões tropicais e subtropicais, e seu ciclo de vida dentro do hospedeiro poder chegar a cinco anos (FIGUEIREDO et al., 2012).

Figueredo et al. (2012) também encontraram ovos dos ancilostomídeos em $19,2 \%$ das 130 amostras coletadas nas quatro estações do ano e foram encontrados 
ovos dessa mesma família em todas as amostras recolhidas em praças de sete escolas diferentes da cidade de Uruguaiana (RS). Nessa mesma pesquisa, foram encontradas quatro amostras positivas das 130 pesquisadas para a presença de Ascaris spp. $(3,1 \%)$ e uma amostra positiva para Trichuris spp. $(0,8 \%)$.

Pinheiro (2016) também encontraram Ascaris lumbricoides, Aspidisca sp., Euplotes sp., Ancylostoma spp., Balantidium sp., Chilomastix mensnili e Trichuris trichiura na areia da praia de Ponta da Fruta, em Vila Velha (ES), e em locais próximos à praia como bancos, castanheiras e saídas de esgoto. Foram realizadas quatro coletas em diferentes datas e somente em uma não foi possível encontrar nenhum tipo de cisto, trofozoítos, ovos ou larvas, com a hipótese de que os dias que antecederam a coleta foram de chuva intensa na região.

Em alguns estudos, como o realizado por Pinheiro (2015), ressalta-se a importância de se ter um controle sobre os animais que habitam locais públicos como praças, praias, locais de convivência como áreas de lazer, pois cães e gatos contribuem para a contaminação ambiental de parasitos com potencial zoonótico. Alves et al. (2014) realizou um estudo sobre a frequência de entreparasitores em fezes de cães coletadas em praças públicas do município de Pindamonhangaba-SP, e também ressalta a importância dos cães como fonte de contaminação em locais públicos por parasitos com potencial zoonótico.

\section{CONCLUSÃO}

Com isso, cabe ressaltar a importância de revitalização do espaço de lazer em que essa pesquisa foi realizada, pois muitas crianças utilizam aquele espaço para brincar e podem estar expostas a contaminação por parasitos com potencial zoonótico, podendo causar doenças que, em casos graves, podem levar a óbito.

Os resultados obtidos sinalizam para a importância e a urgência da ação de órgãos públicos competentes para uma revitalização daquele espaço, assim como uma conscientização da população que o utiliza, além de um controle sobre os animais que habitam aquele espaço. 


\section{REFERÊNCIAS}

ALVES, A. P. S. M.; COELHO, F. A. S.; COELHO, M. D.G. Frequência De Enteroparasitos Em Fezes De Cães Coletadas Em Praças Públicas Do Município De Pindamonhangaba, SP, Brasil. Revista de Patologia Tropical, [s.I.], v. 43, n. 3, p. 341350, 9 out. 2014. Universidade Federal de Goiás. http://dx.doi.org/10.5216/rpt.v43i3.32204. Disponível em: <https://revistas.ufg.br/iptsp/article/viewFile/32204/17235>. Acesso em: 30 jul. 2016.

FIGUEIREDO, M. I. O. et al. Levantamento Sazonal De Parasitos Em Caixas De Areia Nas Escolas Municipais De Educação Infantil Em Uruguaiana, RS, Brasil. Revista de Patologia Tropical, [s.I.], v. 41, n. 1, p.36-46, 30 mar. 2012. Universidade Federal de Goiás. Disponível em: <http://dx.doi.org/10.5216/rpt.v41i1.17744>. Acesso em: 30 jul. 2016.

GASPAR. PREFEITURA DE GASPAR. Perfil. 2014. Disponível em: $<$ http://www.gaspar.sc.gov.br/cms/pagina/ver/codMapaltem/23124>.

Acesso em: 28 jul. 2016.

PINHEIRO, P. Ancilostomose - A. Duodenale E N. Americanus. 2016. Disponível em: <http://www.mdsaude.com/2014/02/ancilostomose.html>. Acesso em: 1 ago. 2016.

PINHEIRO, P. Estrongiloidíase - Strongyloides Stercoralis. 2015. Disponível em: <http://www.mdsaude.com/2013/08/estrongiloidiase.html>. Acesso em: 2 ago. 2016.

PINHEIRO, P. Tricuríase - Trichuris Trichiura. 2016. Disponível em: <http://www.mdsaude.com/2014/02/tricuriase-trichuris-trichiura.html>. Acesso em: 1 ago. 2016.

PORTAL, Colunista (Ed.). Strongyloides sp. - Parasitos. 2013. Disponível em: <http://www.portaleducacao.com.br/farmacia/artigos/45972/strongyloides-spparasitos>. Acesso em: 28 jul. 2016.

REY, L. Parasitologia: parasitos e doenças parasitárias do homem nos trópicos ocidentais. 4 ed. Rio de Janeiro: Guanabara Koogan, 2008.

SILVA, et al. Ocorrência De Parasitos Com Potencial Zoonótico Em Praças E Parques Públicos Da Cidade Do Recife-Pernambuco. In: Biodiversidade E Água: Desafios E Cooperação, 1., 2013, Recife - Pe. Anais... Recife: Universidade Católica de Pernambuco, 2013. v. 2, p. 2 - 12. Disponível em: <http://www.unicap.br/simcbio/wpcontent/uploads/2014/09/OCORRÊNCIA-DE-PARASITOS-COM-POTENCIALZOONÓTICO-EM-PRAÇAS-E-PARQUES-PÚBLICOS-DA-CIDADE-DO-RECIFEPERNAMBUCO.pdf $>$. Acesso em: 30 jul. 2016. 